\title{
IONIC MASS TRANSFER IN FLOWING SOLUTIONS. ELECTROCHEMICAL REACTIONS UNDER IONIC MASS-TRANSFER RATE CONTROL ON CYLINDRICAL ELECTRODES*
}

\author{
J. C. BAzÁn and A. J. Arvía \\ Instituto Superior de Investigaciones, Facultad de Química y Farmacia, and \\ División Ingeniería Química, Facultad de Ciencias Fisicomátematicas, Universidad de \\ La Plata, La Plata, Argentina
}

\begin{abstract}
Several electrochemical reactions under ionic mass-transfer rate-control have been studied on cylindrical electrodes forming part of electrolysis cell. The electrodeposition of copper and the redox reactions for the ferro-ferricyanide systems have been examined, in each case with a large excess of a suitable inert electrolyte. The influences of concentration of the reacting species, rate of flowing, viscosity and diffusivity, height of the working electrode, temperature, and distance between anode and cathode, were studied under streamline-flow conditions. The data are fitted by the dimensionless equation
\end{abstract}

$$
S h=0.525 \cdot e R_{d}^{1 / 2} \cdot S c^{1 / 3} \cdot\left(\frac{h}{d}\right)^{3 / 4} .
$$

The temperature dependence of the kinetic constant yielded the following experimental heats of activation (a) for copper ion deposition $4800 \pm 200 \mathrm{cal} /$ mole (b) for ferricyanide reduction, $3400 \pm$ $200 \mathrm{cal} / \mathrm{mole}$ (c) for ferrocyanide oxidation, $3700 \pm 200 \mathrm{cal} / \mathrm{mole}$

Since the kinetic constant increases when the flow rate is increased and also when the electrode height is decreased, a departure from reversibility has been observed for the redox system reaction when suitable experimental conditions are chosen.

Résumé-On a étudié la vitesse du transport de masse pour le cas d'électrodes cylindriques concentriques, le liquide coulant à travers l'espace compris entre les deux cylindres. On a utilisé diverses réactions d'électrode: Réduction du cuivre, oxydation du ferrocyanure, réduction du ferricyanure. Les paramètres suivants ont été variés: Vitesse d'écoulement de l'ćlectrolyte, concentration et viscosité de la solution, hauteur et diamètre des électrodes cylindriques ainsi que la température. Les résultats peuvent être exprimés à l'aide de la corrélation sans dimensions

$$
S h=0,525 \cdot R e_{d}^{1 / 2} \cdot S c^{1 / 3} \cdot\left(\frac{h}{d}\right)^{8 / 4} \text {. }
$$

La variation de la vitesse de transport avec la température correspond à une énergie d'activation de (a) $4800 \pm 200 \mathrm{cal} / \mathrm{mol}$ pour la réduction du cuivre, (b) $3400 \pm 200$ pour la réduction du ferricyanure et (c) $3700 \pm 200$ pour l'oxydation du ferrocyanure. Comme la vitesse du transport massique augmente lorsque l'écoulement du liquide devient plus rapide et lorsque hauteur diminue on peut observer, dans certaines conditions, des écarts de la réversibilité pour le système redox étudié.

Zusammenfassung-Es wurde die Geschwindigkeit des elektrolytischen Stofftransports an konzentrischen zylindrischen Elektroden untersucht, wobei der Elektrolyt durch den Raum zwischen den Zylindern strömte. Es wurden dabei verschiedene Elektrodenreaktionen verwendet: Kupferabscheidung, Oxydation von Ferrocyanid, Reduktion von Ferricyanid. Variiert wurden: die Durchflussgeschwindigkeit, die Konzentration und die Zähigkeit der Lösung, die Höhe und der Durch messer der zylindrischen Elektroden sowie die Temperatur. Die Ergebnisse können durch folgende dimensionslose Korrelation ausgedrückt werden:

$$
S h=0.525 \cdot R e_{d}^{1 / 2} \cdot S c^{1 / 3} \cdot\left(\frac{h}{d}\right)^{3 / 4} .
$$

Der Temperaturkoeffizient der Stoffübergangszahl entspricht einer Aktivierungswärme von (a) $4800 \pm 200 \mathrm{kal} / \mathrm{Mol}$. für die Kupferabscheidung, (b) $3400 \pm 200$ für die Ferricyanidreduktion und

* Manuscript received 18 June 1963. 
(c) $3700 \pm 200$ für die Ferrocyanidoxydation. Da die Geschwindkgekit des Stofftransports mit zunehmender Strömungsgeschwindigkeit und abnehmender Höhe grösser wird, können unter gewissen Bedingungen Abweichungen von der Reversibilität bei den untersuchten Redoxvorgängen beobachtet werden.

\section{INTRODUCTION}

IN A previous paper ${ }^{1}$ we examined an ionic mass-transfer process by electrolysing copper-sulphate-sulphuric-acid solutions on annular cathodes of different sizes. The results could be correlated in a relatively simple dimensionless equation, and indicated that with streamlined flow, the mass transfer could be considered as taking place on a vertical flat plate with streamline flow along it, the hydrodynamic and diffusion boundary layers having merged. The counter-electrode has no appreciable effect on the profile of the working electrode.

Those results led us to study ionic mass-transfer controlled reactions on inner cylindrical electrodes of a concentric tubular cell, vertically placed. Mass transfer under those conditions has been studied by Putnam et $a .^{2}$ for an electrolysis cell of fixed size. Our previous experience, however, suggested that a fuller study of this problem, with change of the available physicochemical and geometrical variables, would be of interest to obtain the ionic mass-transfer rate equation, and to compare the results with those previously obtained.

Two different types of reactions have been investigated, one in which there is a continuous change of the electrode surface and another involving redox reactions with soluble species respectively the electrodeposition of copper and the redox reactions of ferro-ferricyanide solutions.

The rate equations indicate that both the flow velocity and the height of the working electrode could be adjusted to increase the rate of the diffusion process and therefore, under certain conditions departures from reversibility can be observed in otherwise reversible electrode reactions.

From this fact arises the possibility of using tubular cell arrangements in hydrodynamic voltammetry. This application will be considered in a future publication.

\section{The electrolysis cell}

\section{EXPERIMENTAL}

The electrolysis cell was made with a nickel-plated stainless-steel tube or with a copper tube which was at the same time the non-polarizable electrode of the cell and a part of the hydrodynamic circuit.

Copper and nickel non-polarizable electrodes of diameter of 1.90 and $2.13 \mathrm{~cm}$ respectively were used.

The nickel-plated electrode was especially suitable when alkaline solutions of potassium ferro- and ferricyanide were used, whereas the copper tube was employed with acid copper sulphate solutions.

Working electrodes were made of pure nickel rods, copper plated when used for the electrodeposition of copper.

A diagram of the cell is shown in Fig. 1. A set of seven working electrodes was used, with the dimensions given in Table 1 .

The cylindrical working electrode was axially held into the cell by means of Lucitc rods of the same diameter. The cylindrical metallic pieces were screwed and stuck into cylindrical rods of Lucite and the whole arrangement was finished to the proper diameter and polished on a lathe. Solutions flowed through the annular space formed 


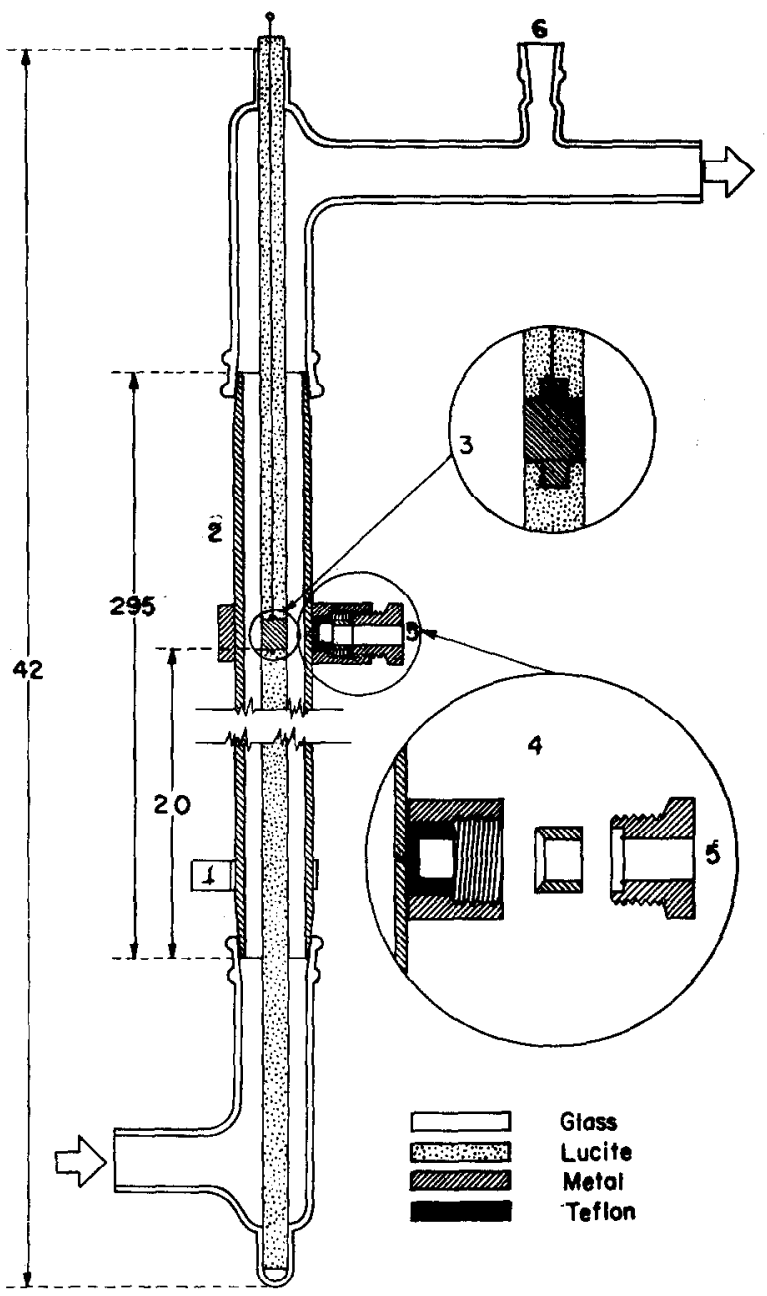

Fig. 1. Electrolysis cell.

1. electrical contact.

2. counter electrode.

3. detail of working electrode.

4. detail of reference electrode joint.

5 . to reference electrode.

6. temperature control.

Lengths in $\mathrm{cm}$.

TABle 1

\begin{tabular}{ccccc}
\hline Electrode & $\begin{array}{c}h \\
\mathrm{~cm}\end{array}$ & $\begin{array}{c}\phi \\
\mathrm{cm}\end{array}$ & $\begin{array}{c}A \\
\mathrm{~cm}^{2}\end{array}$ & $\begin{array}{c}d \\
\mathrm{~cm}\end{array}$ \\
-1 & $1 . \overline{-}$ & -1.00 & $-\frac{3.30}{1 .}$ & -1.88 \\
2 & 4.00 & 1.00 & 12.6 & 1.88 \\
3 & 0.20 & 1.00 & 0.628 & 1.88 \\
4 & 0.80 & 1.00 & 2.51 & 1.88 \\
5 & 0.80 & 1.30 & 3.27 & 1.69 \\
6 & 0.80 & 0.65 & 1.63 & 2.03 \\
7 & 0.08 & 1.00 & 0.215 & 1.88 \\
\hline
\end{tabular}


by the non-polarizable electrode and the insulating rod through a distance of about $30 \mathrm{~cm}$ long before reaching the edge of the working electrode, the actual site where the electrochemical process under diffusion control began to occur. The total length of the electrolytic cell was $47 \mathrm{~cm}$.

The reference electrode was made with platinum wire, copper plated when necessary and immersed in the same solution employed in the cell. It was attached to the external electrode by means of a special metallic holder and the solution was in contact with the flowing liquid through a capillary tube and a pin hole opened on the electrode wall. The reference electrode capillary tip was fixed in the proper position with the aid of Teflon gaskets. It was placed just in front of the middle height of the inner electrode, avoiding any disturbances on the flowing solution.

The rest of the cell was made of Pyrex glass tubing and assembled by means of standard tapered glass joints.

Temperature was read on a calibrated mercury thermometer in the solution immediately beyond the cell.

\section{Flowing solutions}

Two different solutions were studied. The first was an equimolecular solution of potassium ferrocyanide and ferricyanide in nearly $2 \mathrm{M}$ sodium hydroxide. The concentrations of the reacting species were varied from 0.008 to $0 \cdot 1 \mathrm{M}$. These solutions were suitable to study the diffusion process with a reaction where soluble species were involved. For the present case, nickel electrodes were the most suitable.

This redox system has no appreciable activation polarization on nickel electrodes if these are cathodically activated by a hydrogen evolution treatment before assembling the cell. ${ }^{3}$ This was done by placing them in $5 \%$ sodium hydroxide solution at a cathode current density of $\mathrm{ca} 20 \mathrm{~mA} / \mathrm{cm}^{2}$.

The cell had a large surface ratio between the working and the non-polarizable electrodes, making possible independent study of the cathodic and anodic reactions by changing the electrode polarity. The cathodic and anodic reactions are

$$
\mathrm{Fe}(\mathrm{CN})_{6}{ }^{3-}+\mathrm{e}^{-} \rightleftharpoons \mathrm{Fe}(\mathrm{CN})_{6}{ }^{4-} \text {. }
$$

The second system was a solution of copper sulphate in $1.5 \mathrm{M}$ sulphuric acid. The copper ion concentration was varied from 0.008 to $0.223 \mathrm{M}$. Glycerol was added to the copper-sulphate-sulphuric acid-solutions in order to have a range of viscosities from $1.078 \times 10^{-2}$ to $6.130 \times 10^{-2} \mathrm{~cm}^{2} / \mathrm{s}$. The solution was circulated continuously through the cell. Further experimental details and a description of the rest of the arrangement, electrical measuring devices and the procedure are given in our previous publication. ${ }^{1}$

Temperature was varied between 25 and $45^{\circ} \mathrm{C}$, and the flow velocity from 6 to $50 \mathrm{~cm} / \mathrm{s}$.

Purified nitrogen was bubbled continuously during each experiment to eliminate most of the dissolved oxygen, as before.

\section{RESULTS AND I NTERPRETATION}

Typical polarization curves obtained under different conditions are shown in Figs. 2-5. 


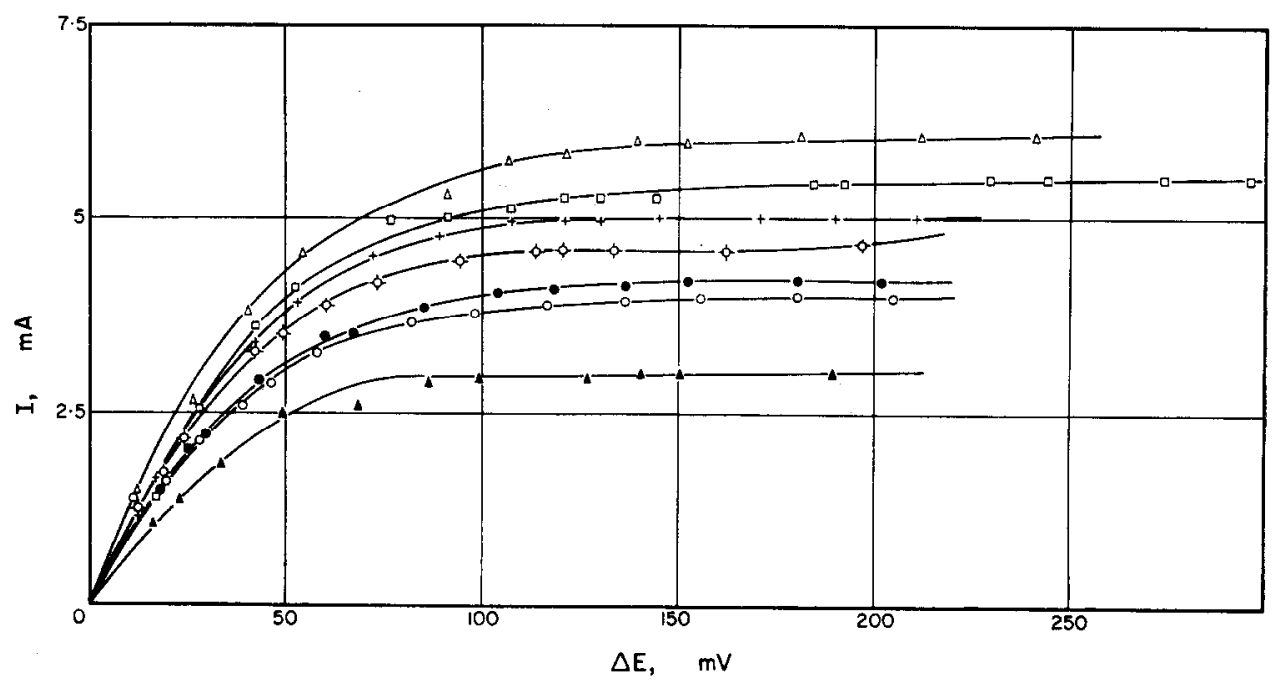

Fig. 2. Current/voltage curves.

Electrode 1. $25^{\circ} \mathrm{C} . \quad C_{0,3}, \quad 0.0111 \mathrm{M}$.

22.0 ; -

$\square: 35 \cdot 9 ; \Delta: 38.9 ; \Delta: 6.4 ; \quad \bigcirc: 18.5 \mathrm{~cm} / \mathrm{s}$

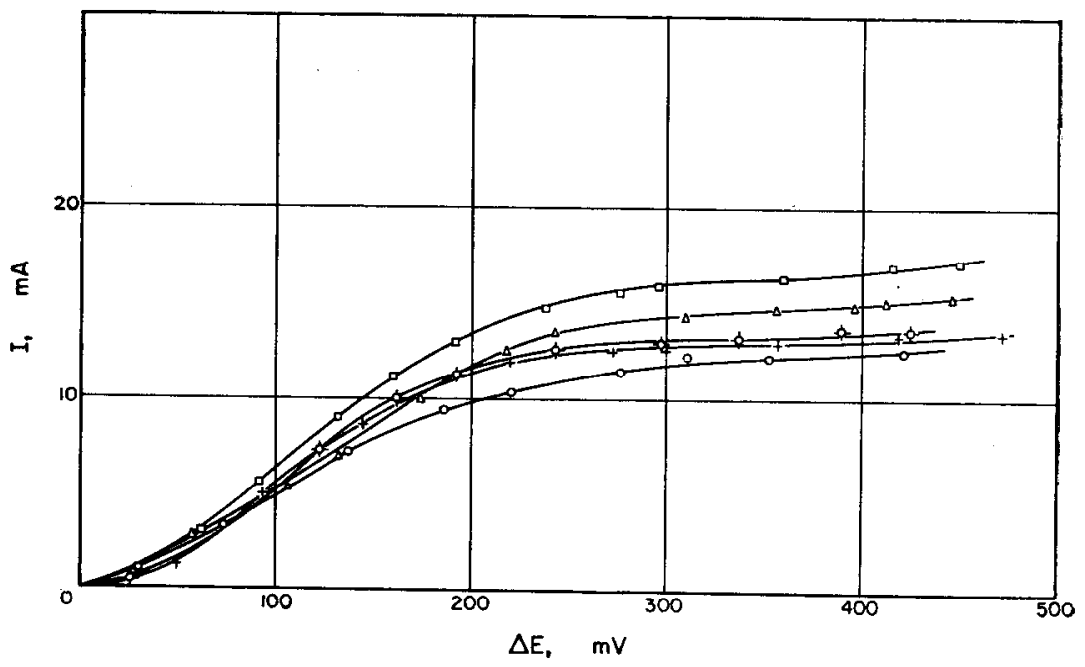

Fig. 3. Current/voltage curves.

Electrode $1.25^{\circ} \mathrm{C} . \quad C_{o}, 0.0480 \mathrm{M} ; \mathrm{C}_{\mathrm{g}}, 5.71 \mathrm{M}$;

$O: 8 \cdot 4 ; \quad+: 20 \cdot 0 ;-\phi-: 24.8$

$\triangle: 30 \cdot 4 ; \square: 37.8 \mathrm{~cm} / \mathrm{s}$ 


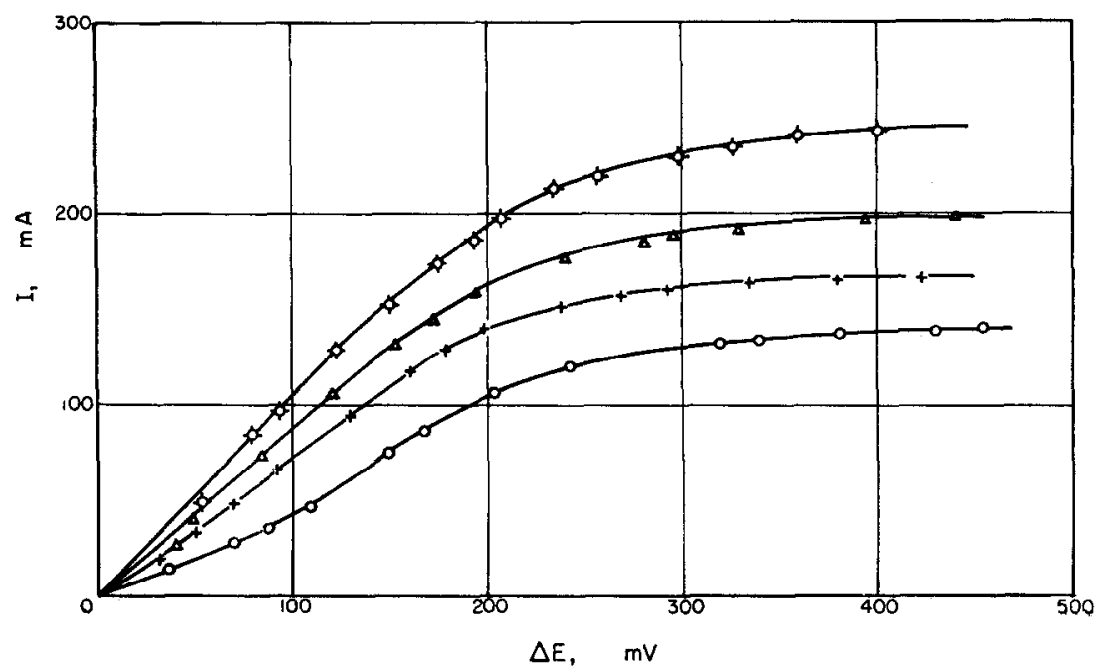

Fig. 4. Current/voltage curves.

Electrode 2. $25^{\circ} \mathrm{C} . \quad C_{0}, 0.0472 \mathrm{M}$;

$\triangle: 37 \cdot 8 ; \quad O: 20 \cdot 0 ; \quad+: 26 \cdot 0 ;-\phi_{-}: 51.7 \mathrm{~cm} / \mathrm{s}$

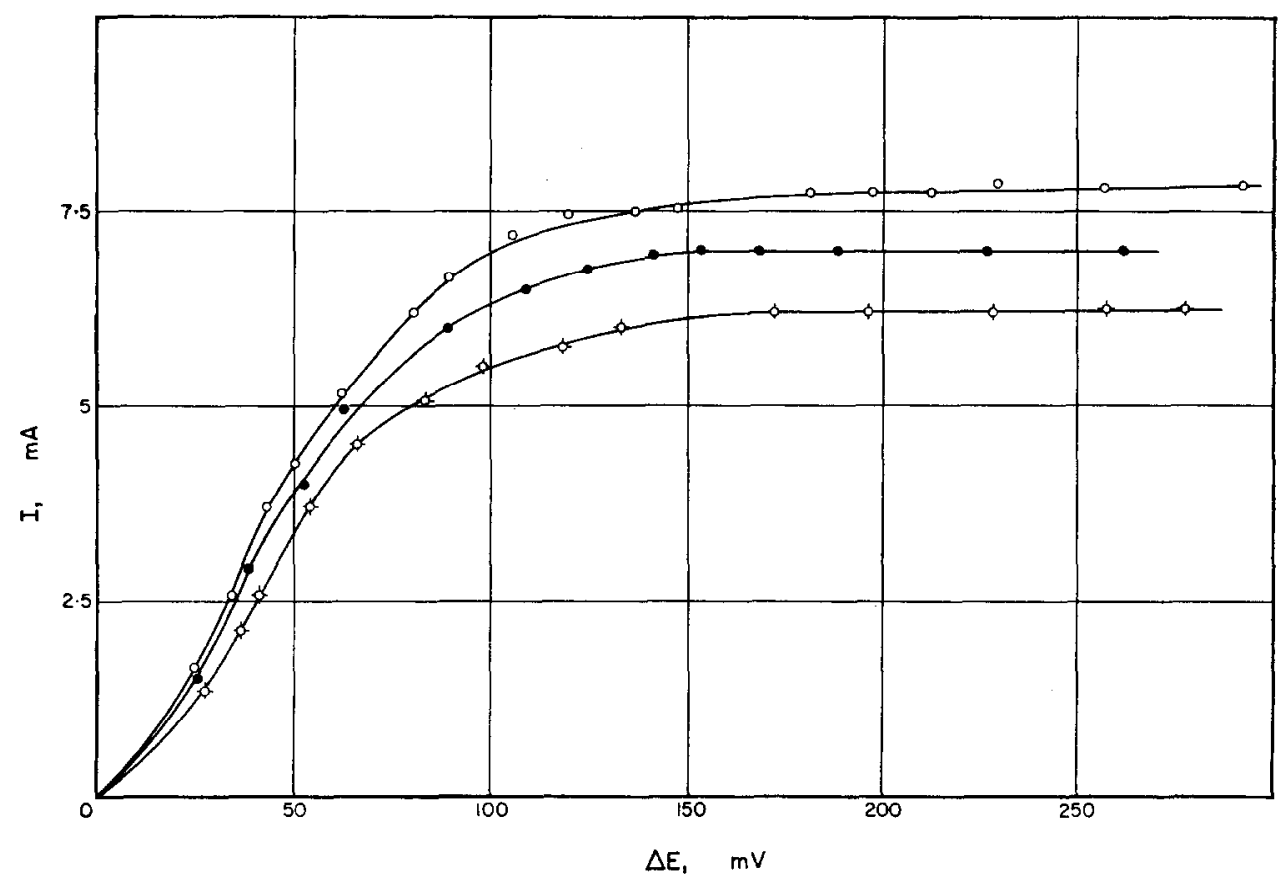

FIG. 5. Current/voltage curves.

Electrode 1. $25^{\circ} \mathrm{C} . \quad C_{0,3}, \quad 0.0113 \mathrm{M}$.

$O: 38.5 ; \quad \bullet: 33.1$; $-C_{-}: 23.9 \mathrm{~cm} / \mathrm{s}$ 
Experimental data are given in Tables 2-6. The following nomenclature is used:

$\mathrm{Nr}$ is the run number; $V$ is the linear mean velocity of the flowing solution in $\mathrm{cm} / \mathrm{s}$, calculated from the total flux and the area of the annular section of the cell; $i_{L}$ is the limiting current density in $\mathrm{mA} / \mathrm{cm}^{2} ; k$ is the mass-transfer kinetic constant in $\mathrm{cm} / \mathrm{s}$, calculated from the current density and the concentration of the diffusing species; $C_{o}, C_{a}, C_{g}, C_{o, 3}, C_{o, 4}$ and $C_{h}$ refer to copper ion, sulphuric acid, glycerol, ferricyanide ion, ferrocyanide ion and sodium hydroxide concentrations respectively, in $\mathrm{mol} / \mathrm{l} ; h, \phi$ and $d$ are the height, diameter and equivalent diameter of the working electrode in $\mathrm{cm} ; \nu$ and $D$ are the average kinematic viscosity and diffusion coefficient of the reacting species, both in $\mathrm{cm}^{2} / \mathrm{s} ; \Delta C_{a}$ and $\Delta C_{h}$ are the changes of concentrations of the supporting electrolytes in mol/l; Sh, $S c$ and $R e$ are the Sherwood, Schmidt and Reynolds numbers respectively. The experimental data shown in the Tables are for $25^{\circ} \mathrm{C}$.

From the temperature dependence of the kinetic constant shown in Figs. 6 and 7 , the experimental temperature coefficients were obtained. The corresponding heats of activation for the different processes are as follows: for copper ion deposition, $4800 \pm 200 \mathrm{cal} / \mathrm{mol}$; for ferricyanide ion reduction, $3400 \pm 200 \mathrm{cal} / \mathrm{mol}$; and for ferrocyanide ion oxidation, $3700 \pm 200 \mathrm{cal} / \mathrm{mol}$.

Table 2. Electrode 1

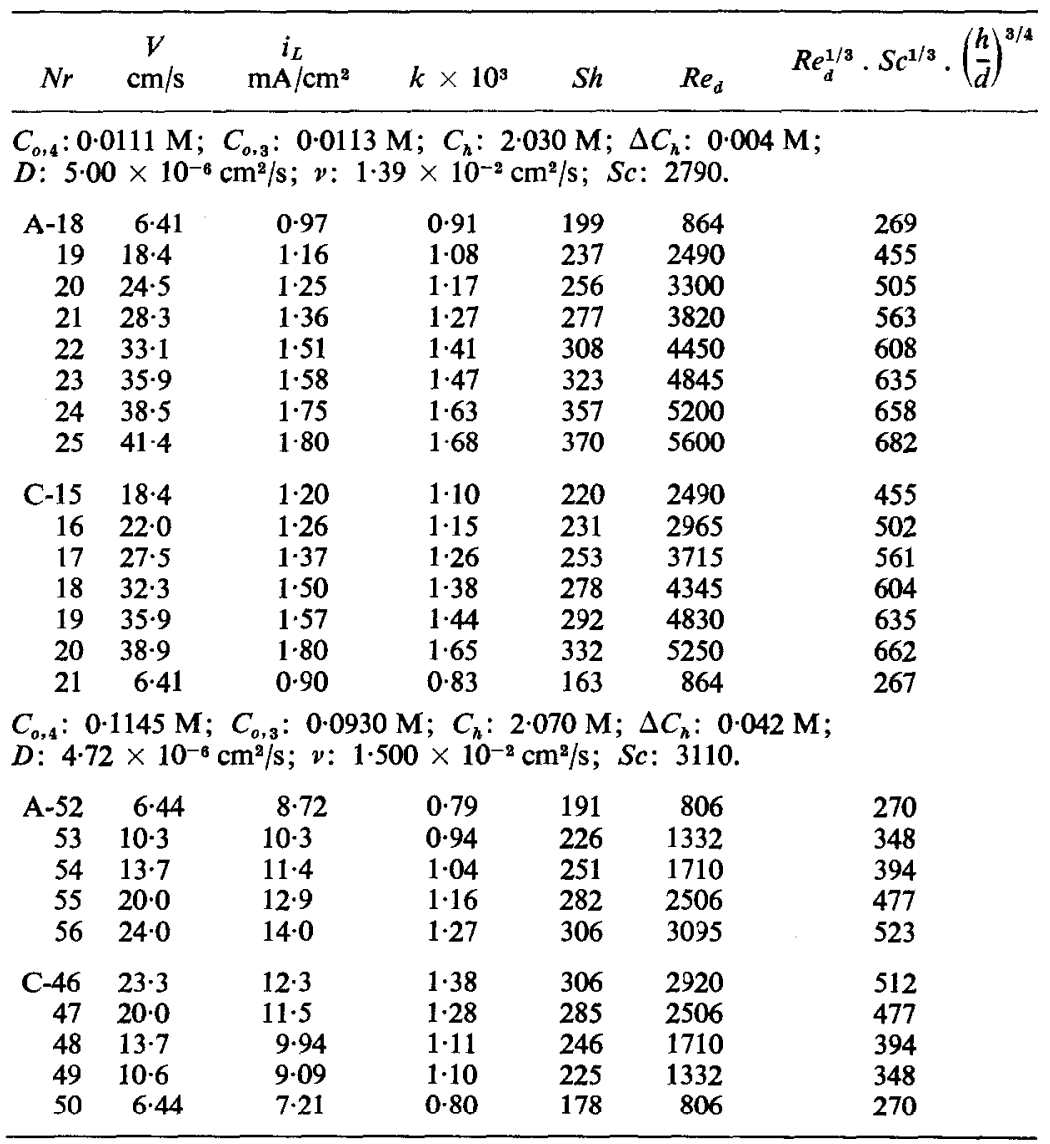


TABLe 3. Electrode 1

\begin{tabular}{|c|c|c|c|c|c|c|}
\hline$N r$ & $\begin{array}{c}V \\
\mathrm{~cm} / \mathrm{s}\end{array}$ & $\begin{array}{c}i_{L} \\
\mathrm{~mA} / \mathrm{cm}^{2}\end{array}$ & $k \times 10^{3}$ & $S h$ & $R e_{d}$ & $R e_{d}^{1 / 2} \cdot S c^{1 / 3} \cdot\left(\frac{h}{d}\right)^{3 / 4}$ \\
\hline \multicolumn{4}{|c|}{$\begin{array}{l}C_{o}: 0.0086 \mathrm{M} ; C_{a}: 1.471 \mathrm{M} ; \Delta C_{a} \\
v: 1.067 \times 10^{-2} \mathrm{~cm}^{2} / \mathrm{s} ; S c: 1684\end{array}$} & \multicolumn{3}{|c|}{$0.004 \mathrm{M} ; D: 6.34 \times 10^{-6} \mathrm{~cm}^{2} / \mathrm{s}$} \\
\hline $\begin{array}{r}\mathrm{Cu}-54 \\
55 \\
56 \\
57 \\
58 \\
59\end{array}$ & $\begin{array}{c}12 \cdot 2 \\
8 \cdot 30 \\
9 \cdot 17 \\
16 \cdot 4 \\
30 \cdot 2 \\
20 \cdot 1\end{array}$ & $\begin{array}{l}2.42 \\
1.88 \\
1 \cdot 92 \\
2 \cdot 61 \\
3 \cdot 15 \\
2 \cdot 69\end{array}$ & $\begin{array}{l}1 \cdot 46 \\
1 \cdot 14 \\
1 \cdot 16 \\
1 \cdot 54 \\
1 \cdot 90 \\
1 \cdot 62\end{array}$ & $\begin{array}{l}242 \\
188 \\
192 \\
260 \\
315 \\
270\end{array}$ & $\begin{array}{l}1846 \\
1256 \\
1388 \\
2490 \\
4580 \\
3047\end{array}$ & $\begin{array}{l}309 \\
256 \\
282 \\
360 \\
487 \\
397\end{array}$ \\
\hline \multicolumn{4}{|c|}{$\begin{array}{l}C_{o}: 0.2230 \mathrm{M} ; \Delta C_{\mathrm{a}}: 1.411 \mathrm{M} ; C_{a} \\
\nu: 1.2140 \times 10^{-2} \mathrm{~cm}^{2} / \mathrm{s} ; S c: 1945\end{array}$} & \multicolumn{3}{|c|}{$0.095 \mathrm{M} ; D: 6.25 \times 10^{-8} \mathrm{~cm}^{2} / \mathrm{s}$} \\
\hline $\begin{array}{r}\mathrm{Cu}-60 \\
61 \\
62 \\
63 \\
64 \\
65\end{array}$ & $\begin{array}{c}8 \cdot 30 \\
9 \cdot 17 \\
12 \cdot 2 \\
16 \cdot 4 \\
30 \cdot 2 \\
20 \cdot 1\end{array}$ & $\begin{array}{l}46 \cdot 7 \\
49 \cdot 4 \\
56 \cdot 9 \\
62 \cdot 7 \\
83 \cdot 1 \\
75 \cdot 7\end{array}$ & $\begin{array}{l}1 \cdot 08 \\
1 \cdot 15 \\
1.32 \\
1.46 \\
1.93 \\
1 \cdot 76\end{array}$ & $\begin{array}{l}183 \\
193 \\
223 \\
245 \\
324 \\
296\end{array}$ & $\begin{array}{l}1103 \\
1218 \\
1622 \\
2185 \\
4025 \\
2678\end{array}$ & $\begin{array}{l}252 \\
264 \\
304 \\
353 \\
480 \\
392\end{array}$ \\
\hline \multicolumn{7}{|c|}{$\begin{array}{l}C_{0}: 0.0472 \mathrm{M} ; C_{a}: 1.420 \mathrm{M} ; \Delta C_{a}: 0.021 \mathrm{M} ; D: 6.25 \times 10^{-6} \mathrm{~cm}^{2} / \mathrm{s} ; \\
\nu: 1.088 \times 10^{-2} \mathrm{~cm}^{2} / \mathrm{s} ; S c: 1596\end{array}$} \\
\hline $\begin{array}{r}\mathrm{Cu}-11 \\
12 \\
13 \\
14\end{array}$ & $\begin{array}{l}20 \cdot 0 \\
37 \cdot 6 \\
51 \cdot 3 \\
28 \cdot 6\end{array}$ & $\begin{array}{l}13 \cdot 3 \\
17 \cdot 7 \\
20 \cdot 3 \\
15 \cdot 5\end{array}$ & $\begin{array}{l}1.45 \\
1.95 \\
2.22 \\
1.70\end{array}$ & $\begin{array}{l}244 \\
327 \\
374 \\
286\end{array}$ & $\begin{array}{l}3000 \\
5635 \\
7700 \\
4280\end{array}$ & $\begin{array}{l}398 \\
545 \\
640 \\
475\end{array}$ \\
\hline \multicolumn{7}{|c|}{$\begin{array}{l}C_{o}: 0.0445 \mathrm{M} ; C_{a}: 1.500 \mathrm{M} ; C_{a}: 3.11 \mathrm{M} ; \Delta C_{a}: 0.008 \mathrm{M} \\
D: 2.85 \times 10^{-6} \mathrm{~cm}^{2} / \mathrm{s} ; \nu: 2.887 \times 10^{-2} \mathrm{~cm}^{2} / \mathrm{s} ; S c: 10120\end{array}$} \\
\hline $\begin{array}{r}\mathrm{Cu}-33 \\
34 \\
35 \\
36\end{array}$ & $\begin{array}{l}20 \cdot 0 \\
28 \cdot 6 \\
37 \cdot 7 \\
51 \cdot 7\end{array}$ & $\begin{array}{l}6 \cdot 52 \\
7 \cdot 12 \\
7 \cdot 88 \\
8 \cdot 80\end{array}$ & $\begin{array}{l}0.76 \\
0.83 \\
0.92 \\
1.02\end{array}$ & $\begin{array}{l}280 \\
306 \\
338 \\
378\end{array}$ & $\begin{array}{l}1117 \\
1598 \\
2105 \\
2891\end{array}$ & $\begin{array}{l}437 \\
477 \\
550 \\
704\end{array}$ \\
\hline \multicolumn{7}{|c|}{$\begin{array}{l}C_{o}: 0.0480 \mathrm{M} ; C_{a}: 1.470 \mathrm{M} ; C_{a}: 5.71 \mathrm{M} ; \Delta C_{a}: 0.006 \mathrm{M} \\
D: 1.45 \times 10^{-8} \mathrm{~cm}^{2} / \mathrm{s} ; v: 6.130 \times 10^{-2} \mathrm{~cm}^{2} / \mathrm{s} ; S c: 42180\end{array}$} \\
\hline $\begin{array}{r}\mathrm{Cu}-44 \\
45 \\
46 \\
47\end{array}$ & $\begin{array}{l}20 \cdot 0 \\
24.7 \\
30 \cdot 4 \\
37 \cdot 7\end{array}$ & $\begin{array}{l}4 \cdot 03 \\
4 \cdot 30 \\
4 \cdot 48 \\
4 \cdot 94\end{array}$ & $\begin{array}{l}0.435 \\
0.464 \\
0.483 \\
0.533\end{array}$ & $\begin{array}{l}314 \\
335 \\
350 \\
385\end{array}$ & $\begin{array}{l}526 \\
648 \\
800 \\
995\end{array}$ & $\begin{array}{l}484 \\
536 \\
595 \\
665\end{array}$ \\
\hline
\end{tabular}

The experiments clearly show that the limiting current density is linearly related to the concentration of the reacting ion as found earlier for annular electrodes.

A general equation for the variables involved in the present study may be expected to be dimensionless, of the form

$$
S h=K \cdot S c^{a} \cdot R e^{b},
$$

as obtained for the tubular electrolysis cell. ${ }^{1}$ In this case the dimensionless numbers are defined as

$$
S h=\frac{k \cdot X}{D}, \quad S c=\frac{v}{D}, \quad R e=\frac{V X}{\nu},
$$

where $X$ is a characteristic length, in $\mathrm{cm}$, chosen according to different criteria analysed later, and $K, a$ and $b$ are constants to be determined experimentally.

A linear relationship was found between the kinetic constant and the square root 
TABLe 4. Electrode 2

\begin{tabular}{|c|c|c|c|c|c|c|}
\hline$N r$ & $\begin{array}{c}V \\
\mathrm{~cm} / \mathrm{s}\end{array}$ & $\underset{\mathrm{mA} / \mathrm{cm}^{2}}{i_{L}}$ & $k \times 10^{8}$ & $S h$ & $R e_{d}$ & $R e_{d}^{1 / 2} \cdot S c^{1 / 8} \cdot\left(\frac{h}{d}\right)^{8 /}$ \\
\hline \multicolumn{7}{|c|}{$\begin{array}{c}C_{0,4}: 0.0111 \mathrm{M} ; C_{0,8}: 0.0113 \mathrm{M} ; C_{\mathrm{h}}: 2.030 \mathrm{M} ; \Delta C_{n}: 0.004 \mathrm{M} ; \\
D: 5.00 \times 10^{-6} \mathrm{~cm}^{2} / \mathrm{s} ; \nu: 1.394 \times 10^{-2} \mathrm{~cm}^{2} / \mathrm{s} ; S c: 2790\end{array}$} \\
\hline $\begin{array}{r}\text { A- }-32 \\
33 \\
34 \\
35 \\
36 \\
37 \\
38\end{array}$ & $\begin{array}{l}16 \cdot 5 \\
23 \cdot 9 \\
28 \cdot 3 \\
33 \cdot 1 \\
38 \cdot 5 \\
41 \cdot 4 \\
47 \cdot 5\end{array}$ & $\begin{array}{l}0.80 \\
0.99 \\
1.07 \\
1.12 \\
1.23 \\
1.29 \\
1.34\end{array}$ & $\begin{array}{l}0.74 \\
0.92 \\
1.00 \\
1.04 \\
1.15 \\
1.20 \\
1.25\end{array}$ & $\begin{array}{r}616 \\
770 \\
837 \\
865 \\
957 \\
1000 \\
1044\end{array}$ & $\begin{array}{l}2230 \\
3320 \\
3820 \\
4450 \\
5195 \\
5600 \\
6410\end{array}$ & $\begin{array}{r}1182 \\
1422 \\
1550 \\
1670 \\
1810 \\
1870 \\
2007\end{array}$ \\
\hline $\begin{array}{r}C-28 \\
29 \\
30 \\
31\end{array}$ & $\begin{array}{l}47 \cdot 5 \\
41 \cdot 4 \\
38 \cdot 5 \\
33 \cdot 1\end{array}$ & $\begin{array}{l}1 \cdot 22 \\
1 \cdot 14 \\
1 \cdot 08 \\
0.98\end{array}$ & $\begin{array}{l}1 \cdot 12 \\
1.04 \\
0.99 \\
0.90\end{array}$ & $\begin{array}{l}868 \\
808 \\
752 \\
687\end{array}$ & $\begin{array}{l}6410 \\
5600 \\
5195 \\
4450\end{array}$ & $\begin{array}{l}2007 \\
1870 \\
1810 \\
1670\end{array}$ \\
\hline \multicolumn{7}{|c|}{$\begin{array}{l}C_{o, 4}: 0.1145 \mathrm{M} ; C_{o, 8}: 0.0930 \mathrm{M} ; C_{h}: 2.070 \mathrm{M} ; \Delta C_{h}: 0.042 \mathrm{M} ; \\
D: 4.72 \times 10^{-8} \mathrm{~cm}^{2} / \mathrm{s} ; \nu: 1.500 \times 10^{-2} \mathrm{~cm}^{2} / \mathrm{s} ; S C: 3110\end{array}$} \\
\hline $\begin{array}{r}\text { A- } 70 \\
71 \\
72\end{array}$ & $\begin{array}{l}10.6 \\
13 \cdot 7 \\
20.0\end{array}$ & $\begin{array}{l}7 \cdot 15 \\
7 \cdot 88 \\
9 \cdot 40\end{array}$ & $\begin{array}{l}0.65 \\
0.72 \\
0.81\end{array}$ & $\begin{array}{l}597 \\
656 \\
754\end{array}$ & $\begin{array}{l}1332 \\
1710 \\
2506\end{array}$ & $\begin{array}{r}960 \\
1088 \\
1315\end{array}$ \\
\hline $\begin{array}{r}C-65 \\
66 \\
67\end{array}$ & $\begin{array}{l}20 \cdot 0 \\
13 \cdot 7 \\
10 \cdot 6\end{array}$ & $\begin{array}{l}7.65 \\
6.71 \\
6.07\end{array}$ & $\begin{array}{l}0.85 \\
0.75 \\
0.68\end{array}$ & $\begin{array}{l}723 \\
634 \\
575\end{array}$ & $\begin{array}{l}2506 \\
1710 \\
1332\end{array}$ & $\begin{array}{r}1315 \\
1088 \\
960\end{array}$ \\
\hline \multicolumn{7}{|c|}{$\begin{array}{l}C_{0}: 0.0472 \mathrm{M} ; C_{a}: 1.420 \mathrm{M} ; \Delta C_{a}: 0.021 \mathrm{M} ; \\
D: 6.25 \times 10^{-6} \mathrm{~cm}^{2} / \mathrm{s} ; v: 1.078 \times 10^{-8} \mathrm{~cm}^{2} / \mathrm{s} ; S c: 1596\end{array}$} \\
\hline $\begin{array}{r}\text { Cu-20 } \\
21 \\
22 \\
23 \\
24\end{array}$ & $\begin{array}{l}37.6 \\
51 \cdot 7 \\
58 \cdot 6 \\
28.6 \\
20 \cdot 0\end{array}$ & $\begin{array}{c}12 \cdot 2 \\
14 \cdot 1 \\
15 \cdot 3 \\
10 \cdot 5 \\
9 \cdot 17\end{array}$ & $\begin{array}{l}1.34 \\
1.55 \\
1.68 \\
1.16 \\
1.01\end{array}$ & $\begin{array}{r}860 \\
990 \\
1075 \\
740 \\
645\end{array}$ & $\begin{array}{l}5635 \\
7750 \\
8780 \\
4280 \\
3000\end{array}$ & $\begin{array}{l}1755 \\
2060 \\
2190 \\
1530 \\
1280\end{array}$ \\
\hline
\end{tabular}

of the mean flow velocity, as shown in Fig. 8 for the different systems. This shows that the exponent $b$ in (1) is $\frac{1}{2}$.

By fixing the values of $V$, the relation of the Sherwood number to the Schmidt number was also determined; the results are shown in Fig. 9 as a plot of $\log S h$ s $\log S c$. The wide range of $S c$ numbers experimentally covered gives sound support to this relationship. The average slope of the straight lines drawn through the experimental point is $\frac{1}{3}$, which is thus the value of exponent $a$ in (1).

The final expression of (1) has been worked out considering two definitions of Reynolds number, taking as characteristic lengths the equivalent diameter of the electrode $d$, which is the diameter corresponding to the annular section of the cell, and the electrode height, $h$ :

$$
R e_{d}=\frac{V . d}{\nu} \text { and } R e_{h}=\frac{V \cdot h}{\nu}
$$

A plot of $S h v s S c^{1 / 3} . R e_{d}^{1 / 2}$ is presented in Fig. 10; it yields different straight lines according to the height of the corresponding electrode. Therefore, a new dimensionless term including the electrode height has to be added to (1). This has been done by plotting $\log S h$ as a function of $\log (h / d)$ for fixed values of $S c^{1 / 3} \cdot R e_{d}^{1 / 2}$, as shown in Fig. 10. The result, shown in Fig. 11, gives $\frac{3}{4}$ for the exponent of the new term of (1). 
TABLe 5. Electrode 3

\begin{tabular}{|c|c|c|c|c|c|c|}
\hline $\mathrm{Nr}$ & $\begin{array}{c}V \\
\mathrm{~cm} / \mathrm{s}\end{array}$ & $\underset{\mathrm{mA} / \mathrm{cm}^{2}}{l_{L}}$ & $k \times 10^{3}$ & $S h$ & $R e_{d}$ & $\operatorname{Re}_{d}^{1 / 2} \cdot S c^{1 / 8} \cdot\left(\frac{h}{d}\right)^{3 / 4}$ \\
\hline \multicolumn{7}{|c|}{$\begin{array}{l}C_{o, 1}: 0.0111 \mathrm{M} ; C_{o, \mathrm{~s}}: 0.0113 \mathrm{M} ; C_{n}: 2.030 \mathrm{M} ; \Delta C_{\mathrm{h}}: 0.004 \mathrm{M} ; \\
D: 5.00 \times 10^{-6} \mathrm{~cm}^{2} / \mathrm{s} ; v: 1.394 \times 10^{-2} \mathrm{~cm}^{2} / \mathrm{s} ; S c: 2790\end{array}$} \\
\hline $\begin{array}{r}\mathrm{C}-34 \\
35 \\
36 \\
37 \\
38 \\
39\end{array}$ & $\begin{array}{l}18 \cdot 5 \\
28 \cdot 3 \\
23 \cdot 1 \\
38 \cdot 5 \\
41 \cdot 4 \\
47 \cdot 5\end{array}$ & $\begin{array}{l}1.59 \\
1.91 \\
1.82 \\
2.04 \\
2.70 \\
3.30\end{array}$ & $\begin{array}{l}1.46 \\
1 \cdot 75 \\
1.68 \\
2 \cdot 19 \\
2 \cdot 48 \\
3.04\end{array}$ & $\begin{array}{r}56 \cdot 7 \\
66 \cdot 8 \\
64 \cdot 0 \\
83 \cdot 5 \\
94 \cdot 6 \\
117\end{array}$ & $\begin{array}{l}2490 \\
3820 \\
4450 \\
5195 \\
5600 \\
6411\end{array}$ & $\begin{array}{l}131 \\
173 \\
176 \\
190 \\
198 \\
213\end{array}$ \\
\hline \multicolumn{7}{|c|}{$\begin{array}{l}C_{C, 4}: 0.1145 \mathrm{M} ; C_{o s}: 0.0930 \mathrm{M} ; C_{h}: 2.070 \mathrm{M} ; \Delta C_{h}: 0.042 \mathrm{M} \\
D: 4.72 \times 10^{-6} \mathrm{~cm}^{2} / \mathrm{s} ; v: 1.500 \times 10^{-2} \mathrm{~cm}^{2} / \mathrm{s} ; S c: 3110\end{array}$} \\
\hline $\begin{array}{r}\text { A-74 } \\
75 \\
76\end{array}$ & $\begin{array}{l}20.0 \\
13 \cdot 7 \\
10.6\end{array}$ & $\begin{array}{l}22 \cdot 3 \\
19 \cdot 1 \\
17 \cdot 6\end{array}$ & $\begin{array}{l}2.01 \\
1.73 \\
1.60\end{array}$ & $\begin{array}{l}92 \cdot 6 \\
79 \cdot 5 \\
73 \cdot 5\end{array}$ & $\begin{array}{l}2506 \\
1710 \\
1332\end{array}$ & $\begin{array}{c}136 \\
113 \\
99 \cdot 5\end{array}$ \\
\hline $\begin{array}{r}C-68 \\
69 \\
70\end{array}$ & $\begin{array}{l}10 \cdot 6 \\
13 \cdot 7 \\
20 \cdot 0\end{array}$ & $\begin{array}{l}14 \cdot 1 \\
15 \cdot 9 \\
17 \cdot 8\end{array}$ & $\begin{array}{l}1.58 \\
1.77 \\
1.98\end{array}$ & $\begin{array}{l}66 \cdot 8 \\
75 \cdot 1 \\
84 \cdot 1\end{array}$ & $\begin{array}{l}1332 \\
1710 \\
2506\end{array}$ & $\begin{array}{l}99 \cdot 5 \\
113 \\
136\end{array}$ \\
\hline \multicolumn{7}{|c|}{$\begin{array}{l}C_{o}: 0.0472 \mathrm{M} ; C_{a}: 1.420 \mathrm{M} ; \Delta C_{a}: 0.021 \mathrm{M} ; \\
D: 6.25 \times 10^{-6} \mathrm{~cm}^{2} / \mathrm{s} ; v: 1.078 \times 10^{-2} \mathrm{~cm}^{2} / \mathrm{s} ; S c: 1596\end{array}$} \\
\hline $\begin{array}{r}\mathrm{Cu}-15 \\
16 \\
17 \\
18 \\
19\end{array}$ & $\begin{array}{l}20 \cdot 0 \\
28 \cdot 6 \\
37 \cdot 6 \\
58 \cdot 6 \\
51 \cdot 7\end{array}$ & $\begin{array}{l}21 \cdot 3 \\
24 \cdot 8 \\
27 \cdot 1 \\
36 \cdot 1 \\
34 \cdot 4\end{array}$ & $\begin{array}{l}2.33 \\
2.72 \\
3.20 \\
3.96 \\
3.77\end{array}$ & $\begin{array}{l}76 \cdot 6 \\
87 \cdot 0 \\
102 \\
127 \\
121\end{array}$ & $\begin{array}{l}3000 \\
4280 \\
5635 \\
8780 \\
7750\end{array}$ & $\begin{array}{l}137 \\
173 \\
187 \\
234 \\
219\end{array}$ \\
\hline
\end{tabular}

Therefore, under the present experimental conditions, the general correlation is

$$
S h=0.525 R e_{d}^{1 / 2} S c^{1 / 3}\left(\frac{h}{d}\right)^{3 / 4},
$$

which is compared with the experimental data in Fig. 12.

The same calculation with the second definition of Reynolds number gives

$$
S h=0.525 R e_{h}^{1 / 2} S c^{1 / 3}\left(\frac{h}{d}\right)^{1 / 4}
$$

Equation (3) gives the following relationships between the formal kinetic constant and the limiting current density and the electrode dimensions and the solution flow properties,

$$
\begin{aligned}
k & =0.525 V^{1 / 2} d^{-1 / 4} h^{1 / 4} D^{2 / 3} v^{-1 / 6}, \\
i_{L} & =0.525 z F C V^{1 / 2} d^{-1 / 4} h^{1 / 4} D^{2 / 3} v^{-1 / 6} .
\end{aligned}
$$

Nernst's definition of the average thickness of the diffusion layer, $\delta_{N}$, is

$$
\delta_{N}=1.90 h^{1 / 4} d^{1 / 4} V^{-1 / 2} D^{1 / 3} v^{1 / 6} .
$$

The maximum error in equations (3) and (4), including the errors involved in all the experimental determinations, is 14 per cent, according to the analysis described previously. ${ }^{1}$ 
Table 6. Electrodes 5, 6 and 7

\begin{tabular}{|c|c|c|c|c|c|c|}
\hline$N r$ & $\begin{array}{c}V \\
\mathrm{~cm} / \mathrm{s}\end{array}$ & $\mathrm{mA}_{L}^{i_{L} / \mathrm{cm}^{2}}$ & $k \times 10^{3}$ & $S h$ & $R e_{d}$ & $\operatorname{Re}_{d}^{1 / 2} S c^{1 / 3}\left(\frac{h}{d}\right)^{3 / 4}$ \\
\hline \multicolumn{7}{|c|}{ Electrode 5} \\
\hline $\begin{array}{r}\mathrm{C}-80 \\
81 \\
82\end{array}$ & $\begin{array}{l}40 \cdot 3 \\
34 \cdot 3 \\
22 \cdot 5\end{array}$ & $\begin{array}{l}14.9 \\
13.4 \\
11.4\end{array}$ & $\begin{array}{l}2.11 \\
1.91 \\
1.61\end{array}$ & $\begin{array}{l}322 \\
291 \\
177\end{array}$ & $\begin{array}{l}4600 \\
3920 \\
2575\end{array}$ & $\begin{array}{l}563 \\
519 \\
419\end{array}$ \\
\hline $\begin{array}{r}\text { A-83 } \\
84 \\
85 \\
86 \\
87 \\
88\end{array}$ & $\begin{array}{l}22 \cdot 5 \\
34 \cdot 3 \\
40 \cdot 3 \\
46 \cdot 8 \\
50 \cdot 4 \\
57 \cdot 8\end{array}$ & $\begin{array}{l}11 \cdot 5 \\
14 \cdot 1 \\
15 \cdot 0 \\
16 \cdot 7 \\
17 \cdot 4 \\
18 \cdot 8\end{array}$ & $\begin{array}{l}1.54 \\
1 \cdot 88 \\
2 \cdot 01 \\
2 \cdot 22 \\
2 \cdot 32 \\
2 \cdot 51\end{array}$ & $\begin{array}{l}279 \\
340 \\
364 \\
403 \\
420 \\
454\end{array}$ & $\begin{array}{l}2575 \\
3920 \\
4600 \\
5340 \\
5750 \\
6595\end{array}$ & $\begin{array}{l}419 \\
519 \\
563 \\
606 \\
529 \\
673\end{array}$ \\
\hline \multicolumn{7}{|c|}{ Electrode 6} \\
\hline $\begin{array}{r}C-83 \\
84 \\
85 \\
86 \\
87 \\
88\end{array}$ & $\begin{array}{l}15 \cdot 6 \\
23 \cdot 8 \\
28 \cdot 0 \\
32 \cdot 5 \\
34 \cdot 9 \\
40 \cdot 1\end{array}$ & $\begin{array}{c}9 \cdot 17 \\
11 \cdot 5 \\
12 \cdot 1 \\
12 \cdot 7 \\
13 \cdot 1 \\
13.9\end{array}$ & $\begin{array}{l}1.30 \\
1.63 \\
1.72 \\
1.82 \\
1.85 \\
1.97\end{array}$ & $\begin{array}{l}199 \\
250 \\
263 \\
279 \\
284 \\
302\end{array}$ & $\begin{array}{l}2145 \\
3270 \\
3840 \\
4470 \\
4800 \\
5510\end{array}$ & $\begin{array}{l}334 \\
412 \\
447 \\
482 \\
492 \\
535\end{array}$ \\
\hline $\begin{array}{r}\text { A-89 } \\
90 \\
91 \\
92 \\
93 \\
94\end{array}$ & $\begin{array}{l}40 \cdot 1 \\
34.9 \\
32 \cdot 5 \\
28 \cdot 0 \\
23 \cdot 8 \\
15 \cdot 6\end{array}$ & $\begin{array}{r}14 \cdot 4 \\
13 \cdot 8 \\
13 \cdot 0 \\
12 \cdot 5 \\
11 \cdot 3 \\
9 \cdot 4\end{array}$ & $\begin{array}{l}1.92 \\
1.83 \\
1.74 \\
1.76 \\
1.51 \\
1.23\end{array}$ & $\begin{array}{l}347 \\
334 \\
315 \\
302 \\
273 \\
\mathbf{2 2 3}\end{array}$ & $\begin{array}{l}5510 \\
4800 \\
4470 \\
3840 \\
3270 \\
2145\end{array}$ & $\begin{array}{l}535 \\
492 \\
482 \\
447 \\
412 \\
334\end{array}$ \\
\hline \multicolumn{7}{|c|}{ Electrode 7} \\
\hline $\begin{array}{r}C-89 \\
90 \\
91 \\
92 \\
93\end{array}$ & $\begin{array}{l}28 \cdot 3 \\
38 \cdot 5 \\
47 \cdot 5 \\
54 \cdot 6 \\
59 \cdot 7\end{array}$ & $\begin{array}{l}23 \cdot 2 \\
26 \cdot 3 \\
29 \cdot 2 \\
32 \cdot 2 \\
32 \cdot 9\end{array}$ & $\begin{array}{l}3 \cdot 27 \\
3 \cdot 72 \\
4 \cdot 12 \\
4 \cdot 56 \\
4 \cdot 66\end{array}$ & $\begin{array}{l}54 \cdot 1 \\
61 \cdot 5 \\
68 \cdot 1 \\
75 \cdot 4 \\
77 \cdot 0\end{array}$ & $\begin{array}{l}3595 \\
4890 \\
6027 \\
6940 \\
7590\end{array}$ & $\begin{array}{c}81 \cdot 0 \\
94 \cdot 4 \\
105 \\
117 \\
123\end{array}$ \\
\hline $\begin{array}{r}\text { A-95 } \\
96 \\
97 \\
98 \\
99\end{array}$ & $\begin{array}{l}28 \cdot 3 \\
38 \cdot 5 \\
47 \cdot 5 \\
50 \cdot 6 \\
54 \cdot 6\end{array}$ & $\begin{array}{l}24 \cdot 6 \\
28 \cdot 2 \\
30 \cdot 2 \\
31 \cdot 6 \\
33 \cdot 6\end{array}$ & $\begin{array}{l}3 \cdot 26 \\
3 \cdot 75 \\
4 \cdot 01 \\
4 \cdot 21 \\
4 \cdot 46\end{array}$ & $\begin{array}{l}53 \cdot 8 \\
61 \cdot 9 \\
66 \cdot 3 \\
69 \cdot 4 \\
73 \cdot 6\end{array}$ & $\begin{array}{l}3595 \\
4890 \\
6027 \\
6430 \\
6940\end{array}$ & $\begin{array}{c}81 \cdot 0 \\
94 \cdot 4 \\
105 \\
108 \\
117\end{array}$ \\
\hline
\end{tabular}

For a more detailed treatment of the results, the change of concentration of the supporting electrolyte next to the electrode surface must be also taken into account. It has been evaluated as

$$
\Delta C_{1}=t_{1} \frac{D_{2}^{2 / 3}}{D_{1}^{2 / 3}} C_{2},
$$

following from (3), where $t_{1}$ is the transport number of species 1 .

Values calculated for $\Delta C_{1}$ are included in the tables. The correction resulting from $\Delta C_{1}$ affects the diffusion coefficients and viscosity in the electrode region. Nevertheless the fluctuations of these data in the literature are higher than the errors involved in the results. 


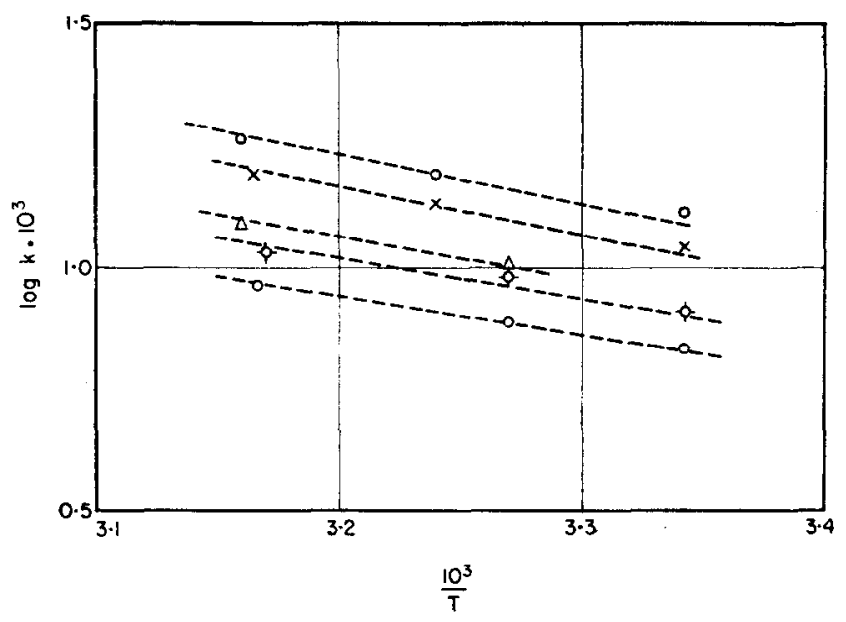

FIG. 6. Arrhenius plot for reduction of ferricyanide ion. (mass-transfer control).

$$
\triangle: 20.0 ;-\phi-: 13 \cdot 7 ; 0: 6 \cdot 4 \text {; (with electrode 1) }
$$

$O: 20.0 ; \times: 10.6 \mathrm{~cm} / \mathrm{s}$; (with electrode 2).

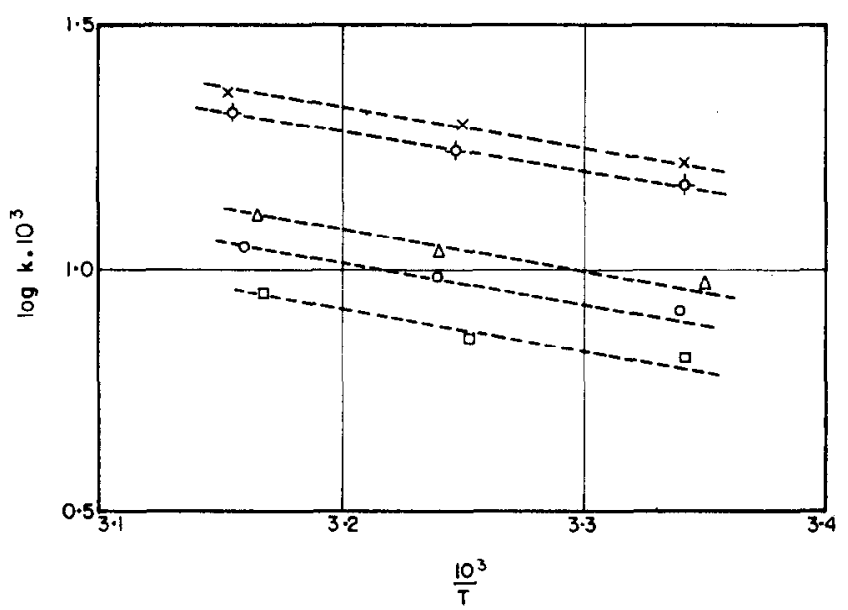

Fig. 7. Arrhenius plot for oxidation of ferrocyanide ion (mass-transfer control).

$$
\triangle: 33 \cdot 3 ; 0: 38 \cdot 5 ; \square: 10 \cdot 6 \text {; (with electrode } 1 \text { ) }
$$
$-\phi-: 10 \cdot 6 ; \quad x: 20.0 \mathrm{~cm} / \mathrm{s}$ (with electrode 2 ).

We have also noticed that the maximum uncertainty in the experiments with the potassium-ferrocyanide-ferricyanide-sodium-hydroxide system is in the ionic diffusion coefficient of the reacting species.

\section{DISCUSSION}

The experimental equations for the ionic mass-transfer process on the inner cylindrical electrodes of the tubular cell have the form of those found for annular electrodes. As is also the case for the rotating disk electrode, ${ }^{5}$ the conical electrode ${ }^{6}$ in flowing solution, and for the streaming mercury electrode, ${ }^{7} k$ and $i_{L}$ are functions of the $-1 / 6$ th power of the kinematic viscosity of the flowing solution and the $2 / 3$ th power of the diffusion coefficient of the species reacting on the electrode. This type of 


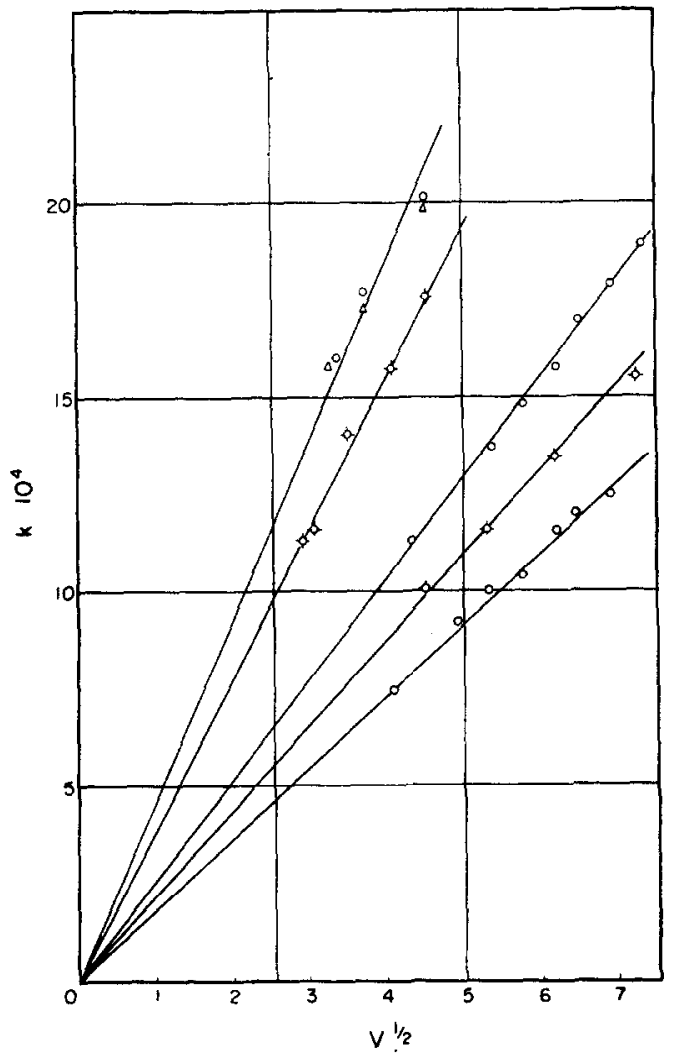

Fig. 8. Plot of $k$ vs $V^{1 / 2}$.

$\triangle:$ reduction of ferricyanide ion.

$O$ : oxidation of ferrocyanide ion.

--: deposition of copper ion.

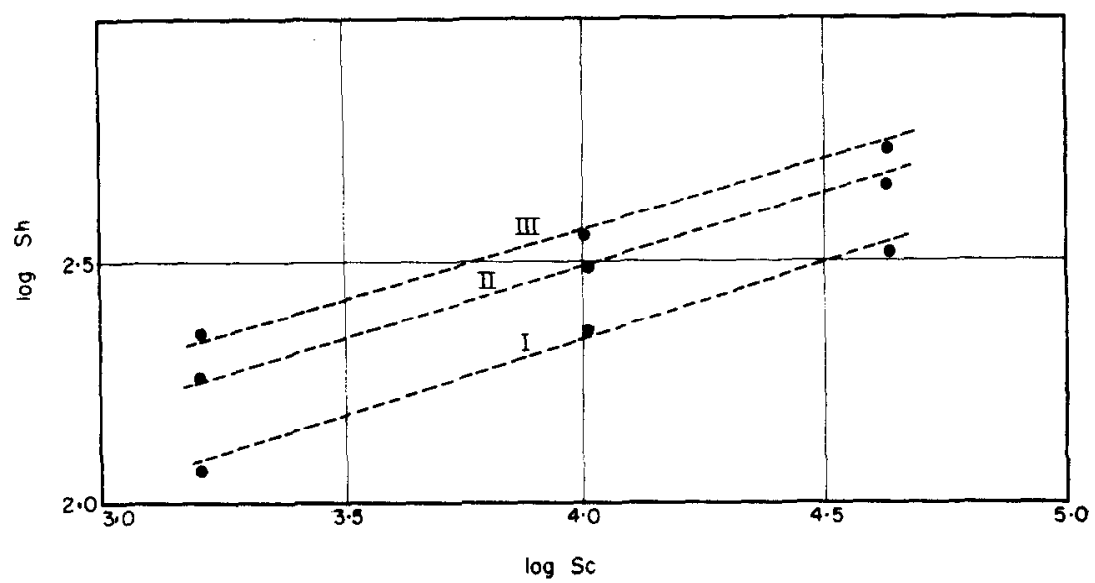

Fig. 9. Plot of $\log S h$ vs $\log S c$.

I, $R e_{d}=625 ;$ II, $R e_{d}=1600 ;$ III, $R e_{d}=2500$. 


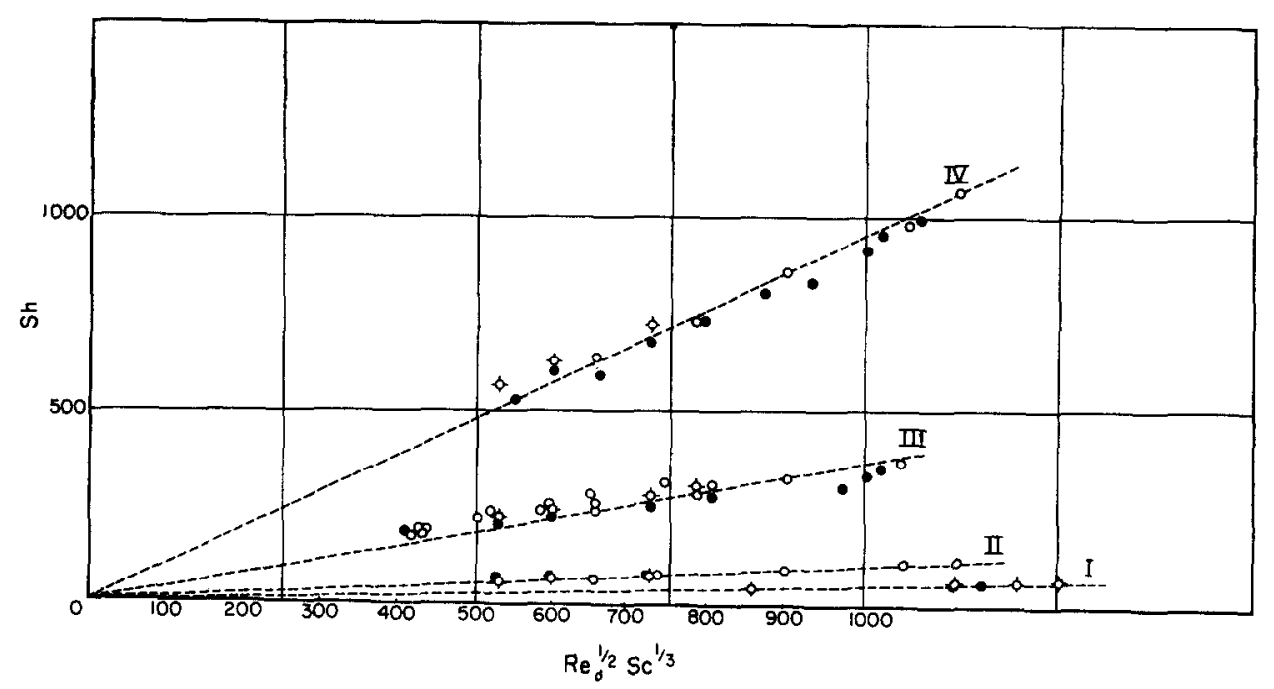

FIG. 10. Plot of $S h v s \operatorname{Re}_{d}^{1 / 2} S c^{1 / 3}$.

I, electrode 7; II, electrode 3; III, electrode 1; IV electrode 2.

๑: oxidation of ferrocyanide ion; - $\phi_{-}^{-}$: reduction ferricyanide ion;

$O$ : deposition of copper ion.

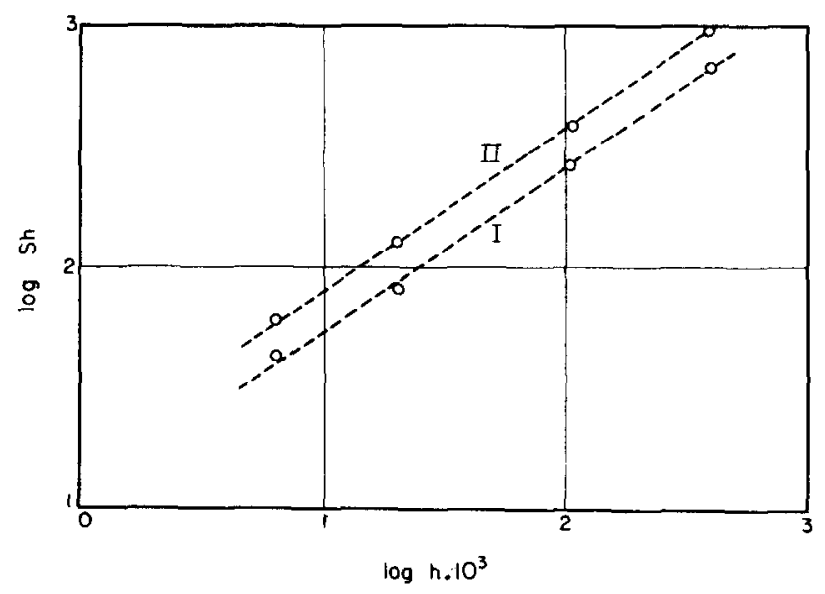

Fig. 11. Plot of $\log S h$ vs $\log h$.

I, $R e^{1 / 2} S c^{1 / 3}=700 ;$ II, $R e_{d}^{1 / 2} S c^{1 / 3}=1000$.

dependence is the one expected when streamline flow occurs on the electrode surface. On the other hand, the ionic mass-transfer mechanism is independent of the electrochemical reactions occurring at the electrodes.

A question arising in the present case concerns the entrance length for a fully developed Poiseuille profile in the annular space. If it is estimated by extending to the present case Schlichting's equation deduced for parallel flat plates, the entrance length would change approximately from 1 to $100 \mathrm{~cm}$, within the flow-rate range of 6-50 $\mathrm{cm} / \mathrm{s}$, for distances between electrodes from 0.83 to $1.48 \mathrm{~cm}$ and kinematic viscosity from $1 \times 10^{-2}$ to $7 \times 10^{-2} \mathrm{~cm}^{2} / \mathrm{s}$ respectively. Therefore, most of the experiments 


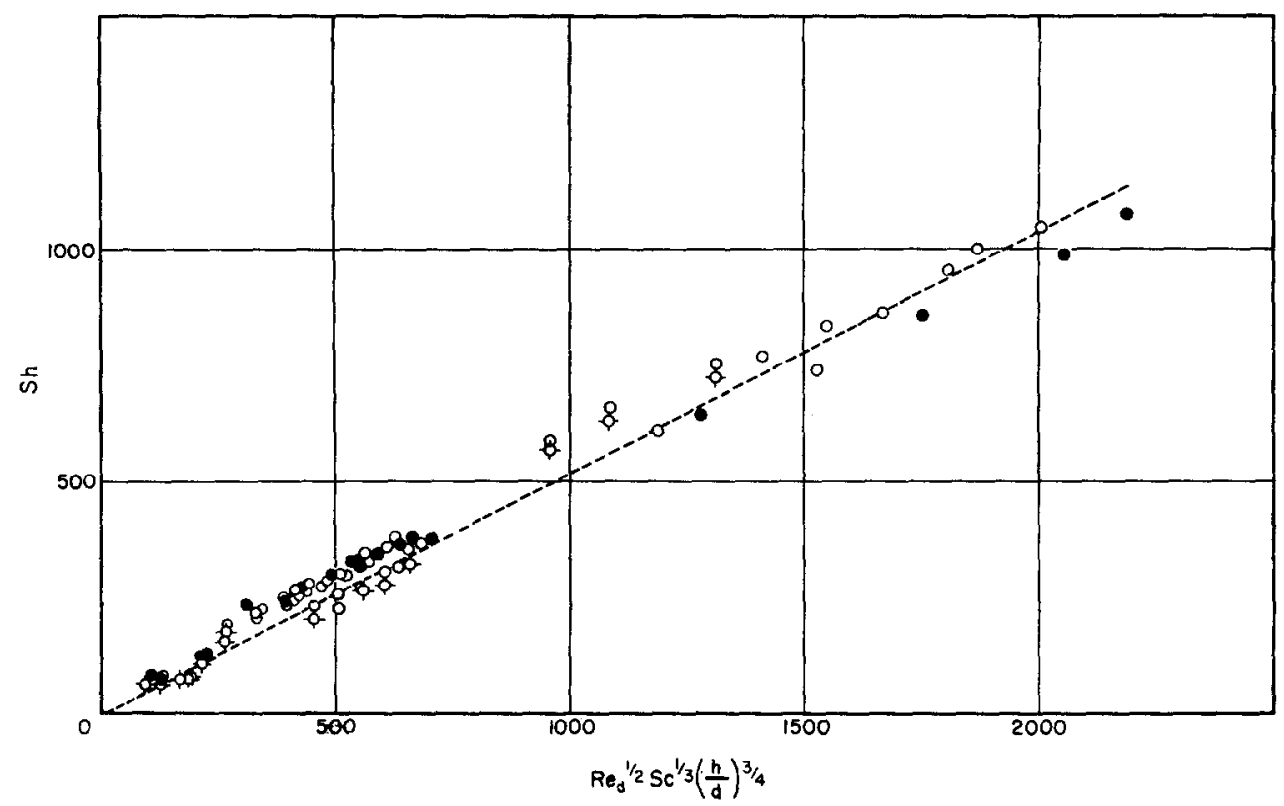

FIG. 12. Plot of equation (3).

-: deposition of copper ion; - - - - reduction of ferricyanide ion;

$O$ : oxidation of ferrocyanide ion.

correspond to a fully developed hydrodynamic profile whereas some of those at the highest rates employed involve a diffusion process taking place within the region of the entrance length.

Nevertheless, the experimental equations found in the present case agree with the concept of a fully developed hydrodynamic profile having been set up when the diffusion process takes place on the electrode. Certainly the situation is much more complicated than that in the case of parallel flat plates, but in spite of this we conclude that the analysis of the mass-transfer process is approximated by the solutions worked out for flat plate electrodes.

If the hydrodynamic boundary layer is not fully developed, one would more reasonably expect an equation of the Lévêque type ${ }^{8}$ to represent the results. Lévêque's equation predicts a linear dependence of $k$ on the cube root of the flow rate. This was not found in the present work.

When equations (3) and (4) are compared to the one previously found for the annular electrode, the existence of a new term expressing a length ratio is observed. This term shows the influence not only of the electrode length but also that of the anode-to-cathode distance, and it means that in the present case the diffusionconvection reaction is actually altered by the existence of the counter-electrode. This probably arises because the velocity profile between the two surfaces of the annulus is not symmetrical, and the radius of zero momentum transfer is a function of the distance between the two surfaces.

Both equations require a linear dependence of the kinetic constant on the square root of the flow rate and on the 1 th power of the electrode height. 


\section{The temperature coefficient of ionic mass transfer in flowing cells}

As discussed elsewhere ${ }^{9,10}$ and as can be deduced from (1) for each particular case, the experimental heat of activation of a transport phenomenon is a complex quantity depending on the heats of activation of diffusion and momentum-transfer processes.

We notice that the heat of activation for the electrodeposition of copper ions on both tubular and annular electrodes are approximately the same, which is a quite reasonable result considering that the kinetic equations for the ionic transfer of the two cells exhibits the same dependence on the diffusion coefficient of the reacting species and on the viscosity of the solution. From (3), the relationship between the experimental heat of activation $E_{T}$, and the heats of activation for the diffusion process, $E_{D}$, and for the momentum-transfer process associated with viscosity, $E_{v}$, is

$$
E_{T}=\frac{2}{3} E_{D}+\frac{1}{8} E_{\nu}
$$

Previously we have observed ${ }^{11}$ that the experimental heat of activation for rotating cylindrical electrodes with turbulent flow can be confounded with the heat of activation for diffusion; $\delta_{N}$ is apparently independent of temperature, as predicted by the Nernst theory ${ }^{12}$ of the diffusion layer. For that case, the coincidence is due to a compensation of the temperature-dependent terms, because since $D$ and $\nu$ depend inversely on $T$, and have the same exponent on the kinetic equation, their effects cancel out.

The temperature coefficient of the diffusion constant for copper ion in coppersulphate-sulphuric-acid solution gives an activation energy for diffusion $E_{D}=$ $5100 \pm 400 \mathrm{cal} / \mathrm{mole}$.

From data obtained in our laboratory, the viscosity dependence with temperature yields $E_{v}=4200 \pm 100 \mathrm{cal} / \mathrm{mole}$. $E_{T}$ obtained with these values of $E_{D}$ and $E_{v}$ substituted in (9) is $4100 \pm 300 \mathrm{cal} / \mathrm{mole}$, almost within the limits of the experimental value of $E_{T}, 4800 \pm 200 \mathrm{cal} / \mathrm{mole}$. Consequently the rate equation satisfies the energy requirements.

Finally, the heat of activation is also independent of the flow rate, as would be expected if the same flow conditions and kinetic equations for mass transfer are obeyed in the region investigated.

Current/voltage curves and concentration polarization for the ferro-ferricyanide couple

Considering (6), the dependence of $k$ on the flow rate and on the electrode height suggests the possibility of increasing the rate of the ionic mass transfer by suitably changing both variables. Thus, a situation could be achieved in which the shape of the current/voltage curves is clearly different from that predicted by pure concentration polarization. For a redox reaction such as that of the ferro-ferricyanide couple, the concentration polarization is given by

$$
\Delta E_{c}=\frac{2 \cdot 3 R T}{Z F} \log \frac{\left(1 \pm I / I_{c}\right)}{\left(1 \top I / I_{a}\right)},
$$

where $I_{c}$ and $I_{a}$ are the cathodic and anodic limiting current densities respectively.

In Fig. 13, current/voltage curves for the ferro-ferricyanide couple under different experimental conditions are plotted according to (10). The overpotentials of the working electrode measured against the reference electrode have been suitably corrected for ohmic drop in the cell. The results give a straight line with slope $2 \cdot 3 R T / F$, and it can be concluded that the redox reaction comes close to thermodynamic reversibility. 


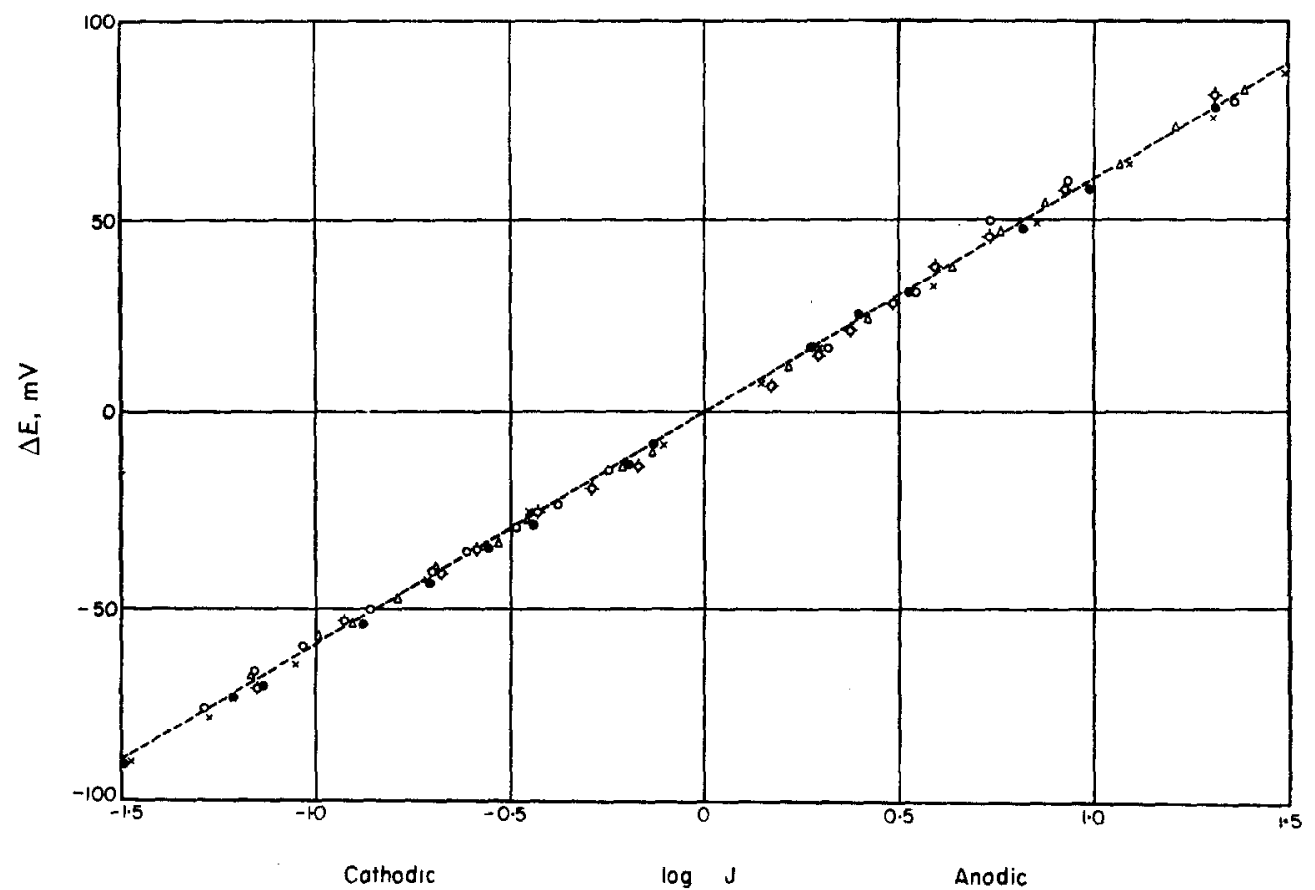

Fig. 13. Test of eq. (10).

Low velocities and electrode 1 .

-: C-47 and A-19; $O$ : C-41 and A-45: $\times$ : C-48 and A-49;

$\Delta: C-42$ and A-54; - - : C-45 and A-55.

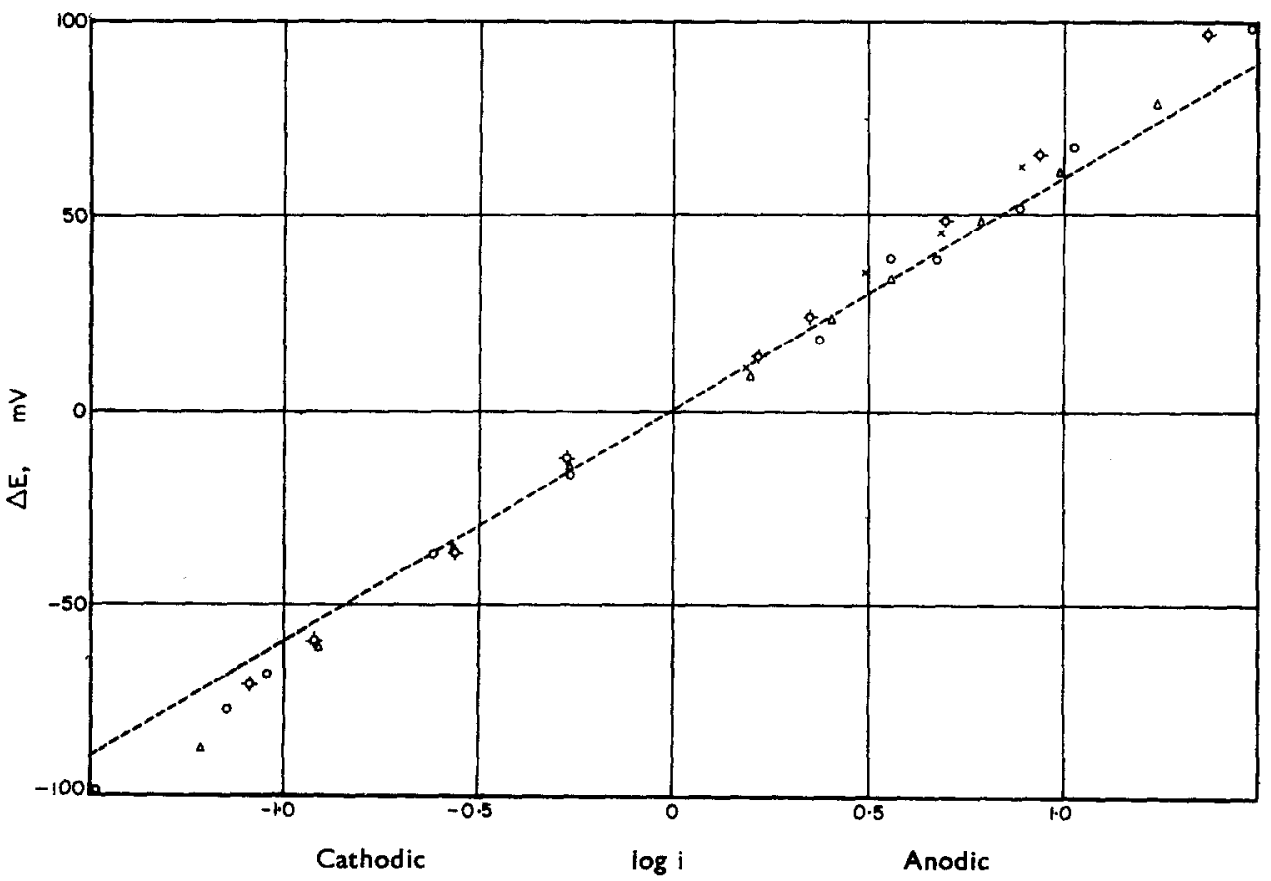

Fig. 14. Test of eq. (10).

High velocities and electrode 7.

$O:$ C-90 and A-95; - - - C-92 and A-98; $\triangle:$ C-93 and A-96; $\times$ : A-99. 
Nevertheless, a deviation from (10) is observed for the same system at the highest flow rate and the smallest electrode height employed in the present work. Fig. 14 shows a departure indicating a larger polarization than that predicted if concentration polarization alone is involved. Therefore, as Petrocelli ${ }^{13}$ has shown for a redox electrode, under such experimental conditions chemical polarization is becoming relatively significant.

Acknowledgements-We thank Prof. Dr. H. J. Schumacher for his interest in this work. The present work was supported with funds and a fellowship (J.C. B.) by the Consejo Nacional de Investigaciones Científicas y Técnicas of Argentina.

\section{REFERENCES}

1. J. C. BazÁn and A. J. Arvía, Electrochim. Acta 9, 17 (1964).

2. C. S. Lin, E. B. Denton, H. S. GASkill and L. G. Putnam, Industr. Engng Chem. 43, 2136 (1951).

3. M. Eisenberg, C. W. Tobias and C. R. Wilke, Trans. Electrochem. Soc. 101, 306 (1954).

4. A. R. Gordon and A. Cole, J. Phys. Chem. 40, 733 (1936).

5. B. LeVICH, Disc. Faraday Soc. 1, 37 (1947).

6. J. JoRdan and R. A. JAvick, J. Amer. Chem. Soc. 80, 1264 (1958).

7. A. Ruus, J. Llopis and S. Polo, An. Fis. Quim. 45, 1039 (1949).

8. P. LévêQue, J. Chim. Phys. 49, 266 (1952).

9. L. L. Bircumshaw and A. C. Riddiford, Quart. Rev. Chem. Soc., Lond. 6, 157 (1952).

10. B. LeVICH, Physicochemical Hydrodynamics. Prentice-Hall, Englewood, N.J. (1962).

11. A. J. Arvía and J. S. W. CarrozzA, Electrochim. Acta 7, 65 (1962).

12. W. NERNST, Z. phys. Chem. 47, 53 (1904).

13. J. V. Petrocelli, Trans. Electrochem. Soc. 98, 187 (1951). 